\title{
Control inteligente de un robot manipulador para colocación de objetos
}

\author{
Fausto Freire Carrera \\ Universidad Tecnológica Equinoccial \\ ffreire@ute.edu.ec \\ Milton Molina Cadena \\ Universidad Tecnológica Equinoccial \\ xav.1@hotmail.com \\ Olga Chadrina \\ Universidad Tecnológica Equinoccial \\ olga.chadrina@ute.edu.ec
}

Recibido: 01 de septiembre / Aprobado: 30 de noviembre 2017

\section{Resumen}

En este artículo se presenta los resultados de la implementación de un sistema informático que controla un robot manipulador Mitsubishi Melfa RV2AJ, para identificar figuras geométricas de color rojo, recoger y colocar en sus contenedores correspondientes, utilizando visión artificial, con parámetros del entorno controlados. El reconocimiento de objetos y asociación con un contenedor específico se realizó utilizando elementos de inteligencia artificial, y las herramientas de desarrollo utilizadas fueron OpenCV y Qt Creator. Los resultados obtenidos de aciertos de reconocimiento y colocación de los objetos en los contenedores fueron del $98,25 \%$, existiendo un error del $1,75 \%$ debido a la similitud entre los objetos de forma circular y octangular.

Palabras clave: manipulador, robótica, reconocimiento, visión artificial. 


\section{Abstract}

This article presents the results of the implementation of a computer system that controls a manipulating robot Mitsubishi Melfa RV2AJ, which identifies, collects and place red geometric figures in their corresponding containers using artificial vision with controlled environmental parameters. Object recognition and association with a specific container was performed using artificial intelligence elements, and the development tools used were OpenCV and Qt Creator. The results obtained from successful recognition and positioning of the objects in the containers were $98.25 \%$, with an error of $1.75 \%$ due to the similarity between circular and octagonal objects.

Keywords: manipulator, robotics, recognition, artificial vision. 


\section{Introducción}

$\mathrm{L}$

os avances de la inteligencia artificial aplicados a ingeniería en la actualidad son impresionantes, en parte debido al incremento del grado de complejidad de los algoritmos, herramientas y sistemas de cómputo que se utilizan.

La amplia utilización de manipuladores robóticos en la industria cada año se incrementa. Según la Federación Internacional de Robótica (FIR) el ańo 2015 se vendieron 248.000 unidades que representa un incremento del $12 \%$ con respecto al año anterior, lo que supone un auge mundial en la automatización, se espera que para el 2018 unos 2,3 millones de unidades entren en funcionamiento (FIR, 2016).

Los avances en las tecnologías de la información, enfocados a internet de las cosas, dará como resultado la aparición de fábricas pequeñas e inteligentes que permitan alcanzar altos estándares de producción flexible y rentable, esto obliga a que se incorporen sistemas de visión artificial, que garanticen una producción de calidad y niveles de seguridad, por esta razón surge la necesidad de integrar la visión artificial, con sistemas de control inteligente a los manipuladores robóticos.

Los sistemas de visión artificial permiten analizar, identificar y procesar, gran cantidad de información que pertenece al entorno de trabajo del manipulador, la información que se extrae corresponden a propiedades geométricas y tipos de materiales de los objetos, esto sin la necesidad de integrar costosos dispositivos adicionales, lo cual permite que los manipuladores puedan interactuar con el entorno y los objetos (Gómez-Allende, 1994).

En esta investigación la posición del efector final del manipulador se determina mediante el análisis de las imágenes obtenidas por una cámara acoplada a un extremo del manipulador.

Este tipo de control inteligente puede ser utilizado sobre líneas de producción en las que se requiera implementar un sistema de calidad o de clasificación.

La implementación de sistemas de control visual, por ejemplo en una industria, permitiría agilizar procesos, aumentar la productividad y reducir costes, dependiendo de la función que cumpla el sistema implementado.

\section{Materiales y métodos}

La metodología mecatrónica fue implementada para el desarrollo del trabajo y se compone de los siguientes elementos: análisis de requerimientos, desarrollo de los diseños, prototipo virtual, prototipo físico, pruebas y mantenimiento del sistema.

Para cumplir con los requerimientos del proyecto fue necesario considerar los siguientes aspectos:

El programa de reconocimiento artificial debe adquirir y clasificar la imagen del objeto acorde a su geometría, además de traducir la posición inicial y la final donde debe ser colocado el objeto en coordenadas físicas reales. 
- La traducción de pixeles a una cantidad de medida real debe regirse por una resolución que permita tener una posición exacta del objeto, minimizando el error al trasladarlo de una posición a otra.

- El sistema de control para el posicionamiento del manipulador debe realizar la conversión de las coordenadas adquiridas por visión artificial en posiciones angulares para cada articulación como requerimiento para efectuar la cinemática de 5 grados de libertad presentes en el brazo robótico.

\section{Robot manipulador}

En la figura 1 se observa el robot angular de brazo articulado Melfa RV2AJ, diseñado para la manipulación y transporte de objetos. La estructura está compuesta de elementos rígidos conectados por uniones de rotación en una cadena cinemática abierta, la carga máxima en el extremo final es de $2 \mathrm{~kg}$.

La estructura está compuesta por servomotores de corriente alterna que realimentan al sistema mediante codificadores de posición absoluta, lo que permite a la controladora conocer la velocidad de desplazamiento y la posición de cada articulación presentando una variación de $\pm 0.02 \mathrm{~mm}$ en la precisión final de todo el sistema.

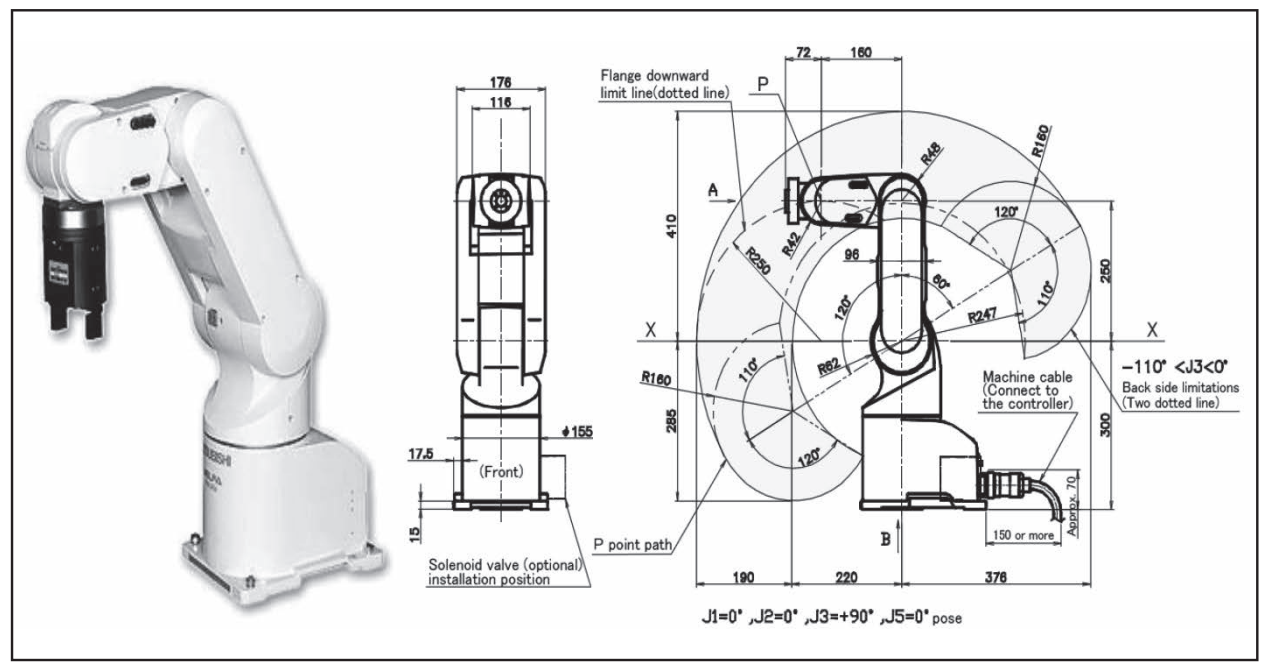

Figura 1. Robot Melfa RV2AJ (Mitsubishi Electric, 2001).

Las características del manipulador melfa RV2AJ permiten que tenga un rango de trabajo amplio, lo cual es necesario en este proyecto para posicionar el efector final a una altura adecuada para determinar la forma del objeto y la posición donde este será colocado.

Para este proyecto no se considera la velocidad de traslación del objeto como una variable requerida, únicamente se evidencia la eficiencia de traslación y la precisión para colocar el objeto en una posición final. 
Para efectuar el movimiento del manipulador y controlar la posición y la orientación del efector final, se utilizó la cinemática inversa (resolución geométrica) figura 2, que permitió encontrar el valor angular para cada articulación, partiendo del conocimiento de las coordenadas donde debía ubicarse el efector final del manipulador.

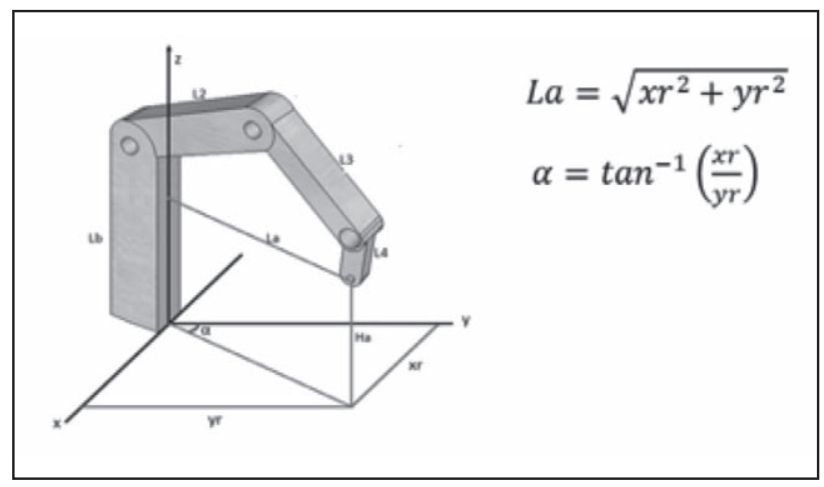

Figura 2. Ubicación espacial efector final.

Para obtener los valores de xr, yr se utilizó visión artificial y de esta manera reconocer la forma del contenedor del objeto y adquirir las coordenadas del centro. Esto convierte a la cámara colocada en el extremo del robot en un sensor de posicionamiento, de las imágenes capturadas se realiza la traducción de pixeles a medidas reales obteniendo así las coordenadas para posicionar el efector del robot

Para realizar el cálculo geométrico que describa la cinemática del manipulador se considera que el movimiento se restringe a la posición que muestra la figura 3, es decir las articulaciones se encuentran en un rango angular positivo, porque la tarea que realiza el robot es manipular objetos en el plano (x,y).

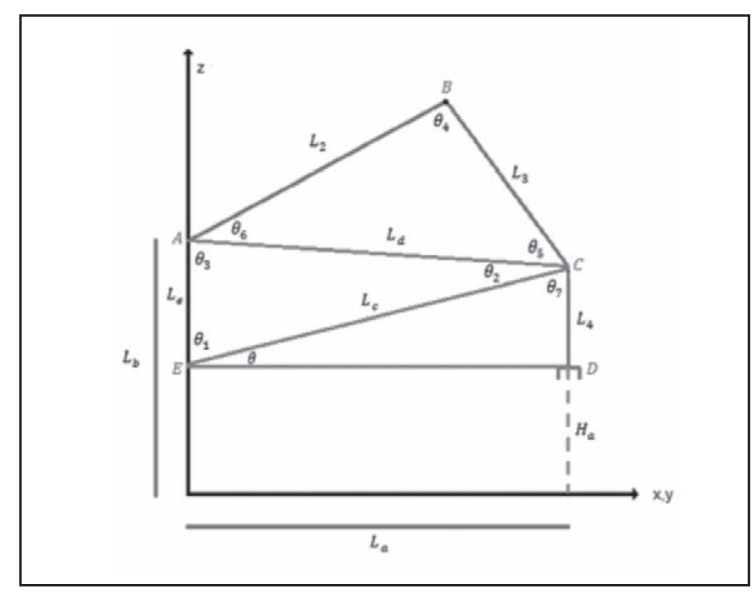

Figura 3. Configuración geométrica de movimiento. 
La solución geométrica encontrada es válida siempre que el ángulo $\theta \_3$ sea menor de 90 grados, en caso de ser diferente la ecuación empleada para la resolución del triángulo (A, C, E) sería errónea, dando como resultado valores angulares para la cinemática diferentes a los esperados, es necesario considerar que el extremo final del manipulador siempre cumplirá la condición de perpendicularidad hacia el plano $(\mathrm{x}, \mathrm{y})$, porque al instante de colocar el objeto se requiere que este sea paralelo con la superficie del contenedor. Mediante la caracterización de los triángulos que conforman la geometría del manipulador se obtiene como resultado el valor angular de cada articulación con lo cual se puede acceder a una posición cinemática que cumpla los requerimientos del proyecto en cuanto precisión para la colocación de los objetos.

Como se muestra en la figura 4, finalmente se obtiene las posiciones angulares para cada articulación deducida por el complemento existente entre el eje de movimiento y los ángulos $\theta$ anteriormente ya calculados.

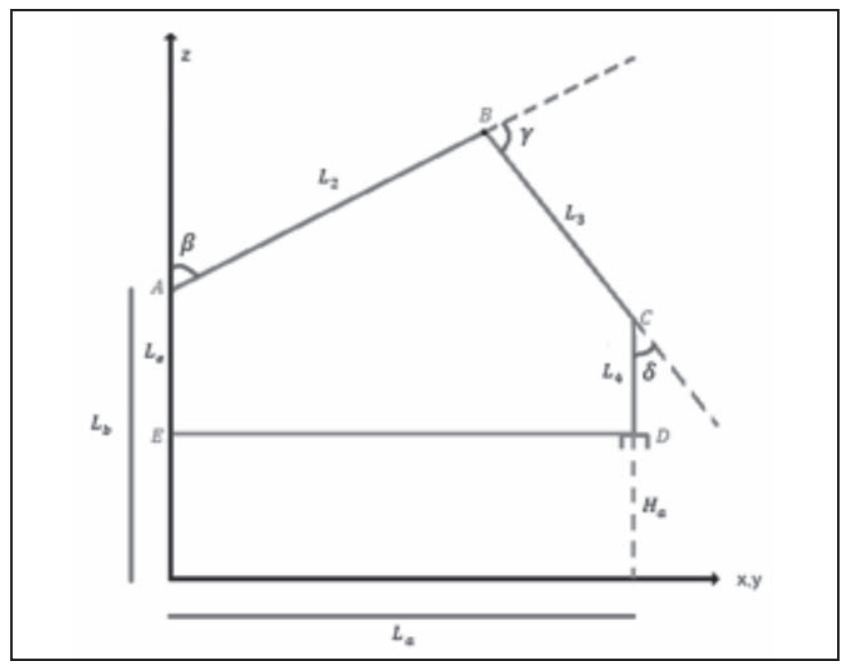

Figura 4. Posición angular final.

\section{Procesamiento de las imágenes}

La entrada al sistema de visión artificial es una imagen obtenida por una cámara digital, mientras que la salida es una serie comandos, para que el robot pueda interactuar en tiempo real con el entorno y se adapte a los cambios que se presenten.

Para el procesamiento de imágenes se realizaron los siguientes pasos:

- Conversión de RGB a HSV

- Umbralizacion de imagen HSV

- Binarizacion de la imagen

- Erosión y dilatación de la imagen

- Segmentación 


\section{Reconocimiento de formas}

En esta etapa ingresan dos imágenes preprocesadas las cuales contienen por separado la información de la forma del objeto que será manipulado por el robot y las formas de los contendores destino, figura 5.

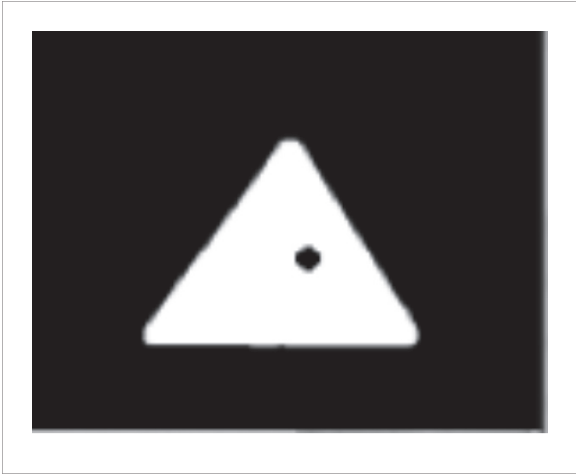

Figura 5a. Objeto a ser manipulado.

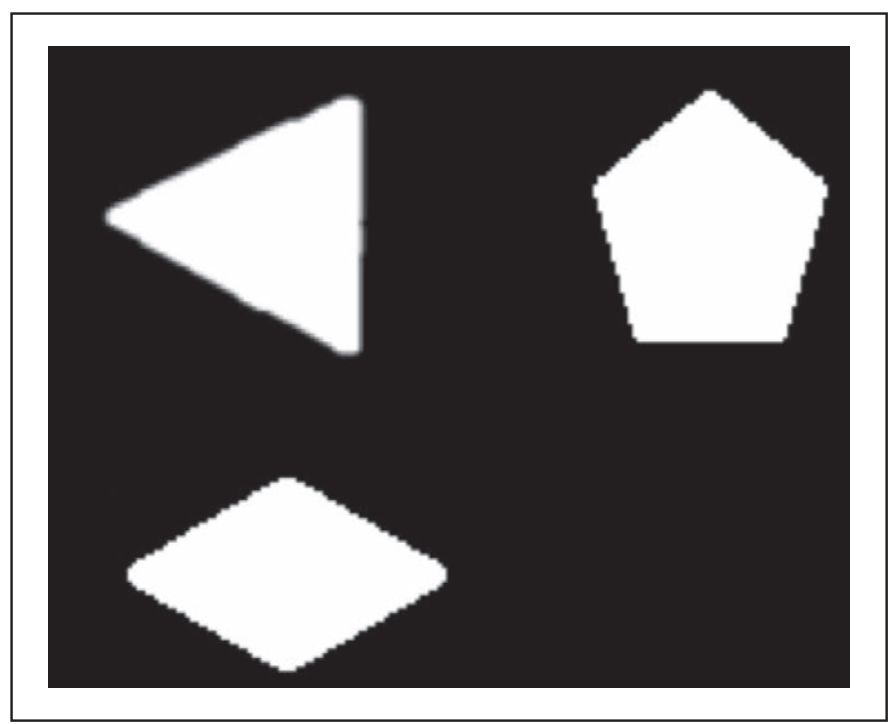

Figura 5b. Contenedor.

Posteriormente se extraen los momentos invariantes de Hu de cada imagen, para encontrar los parámetros propios correspondientes a las geometrías que describen a cada uno.

Los momentos invariantes pueden ser considerados como un promedio ponderado de los pixeles de una imagen, a partir de los momentos geométricos que presentan características de varianza ante la rotación y escalamiento. 
Donde $\mu \_p q$ es el momento geométrico de orden $(\mathrm{p}+\mathrm{q}), \mathrm{f}(\mathrm{x}, \mathrm{y})$ es el valor del pixel en la posición $(\mathrm{x}, \mathrm{y})$ de la imagen $\mathrm{y}(\overline{\mathrm{x}}, \overline{\mathrm{y}})$ es el centroide de la misma. A partir de estos momentos se obtiene un momento de orden $(p+q)$ que es invariante al escalamiento. $\mathrm{Al}$ obtener como resultado una matriz en la que consta los momentos para todas las imágenes segmentadas se realiza una diferencia ponderada para elegir qué valor es el más cercano al objeto a manipular, de esta manera se obtiene un solo valor porcentual próximo que relaciona dos geometrías cumpliendo con la asociación de objetos.

$$
n_{p q}=\frac{\mu_{p q}}{\mu_{00}^{1+\frac{p+q}{2}}}
$$

En la figura 6a, se puede apreciar la imagen del contenedor y del objeto (triángulo con punto en el centro) a ser colocado; en la figura $6 \mathrm{~b}$, se puede apreciar el reconocimiento que realiza el sistema definiendo y la forma y posición en el contenedor.

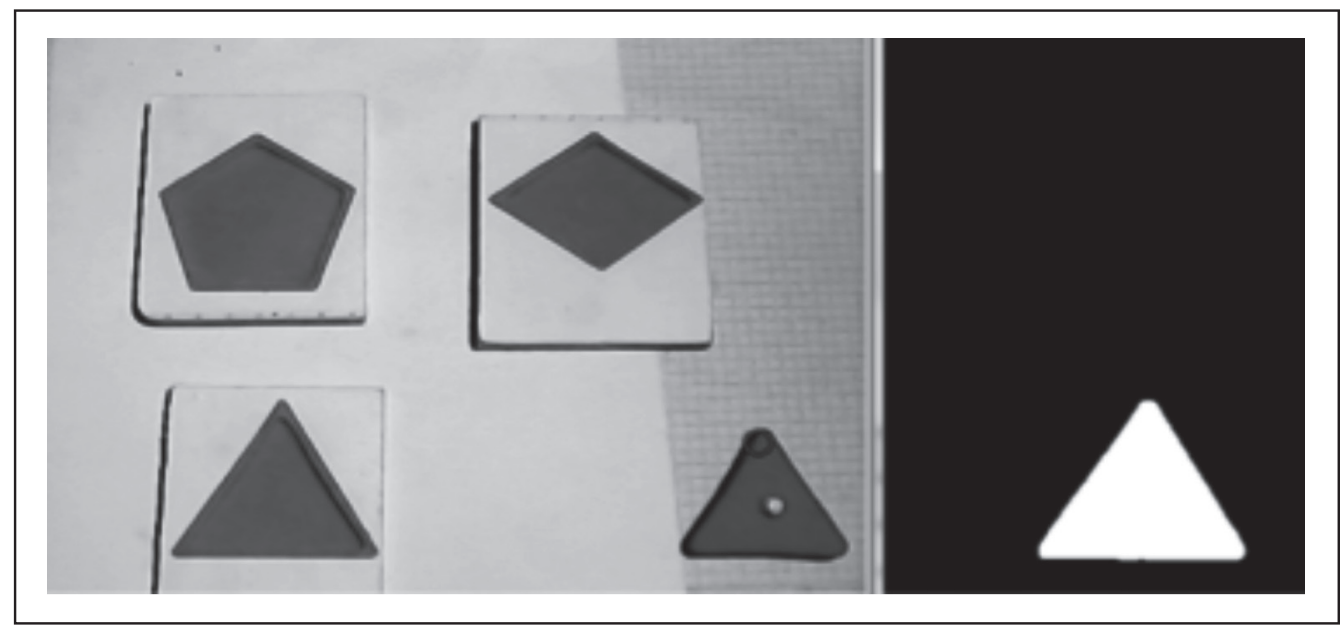

Figura 6a.

Figura $6 b$.

\section{Resultados}

En la tabla 1 se presenta los resultados del reconocimiento con diferentes objetos. Existe un error de reconocimiento correspondiente al 1,75\%, entre los objetos de forma circular y los de forma octangular, es decir aproximadamnete un error por cada 14 aciertos, lo cual demuestra la efectividad del proceso propuesto, con los demás objetos el reconocimiento es del 100\%. 
Tabla 1. Resultado de pruebas de reconocimiento

\begin{tabular}{|c|c|c|}
\hline $\begin{array}{c}\text { Formas } \\
\text { geométricas }\end{array}$ & Intentos & Aciertos \\
\hline Círculo & 15 & $93 \%$ \\
\hline Óvalo & 15 & $100 \%$ \\
\hline Triángulo & 15 & $100 \%$ \\
\hline Cuadrado & 15 & $100 \%$ \\
\hline Rectángulo & 15 & $100 \%$ \\
\hline Rombo & 15 & $100 \%$ \\
\hline Pentágono & 15 & $100 \%$ \\
\hline Octágono & 15 & $93 \%$ \\
\hline
\end{tabular}

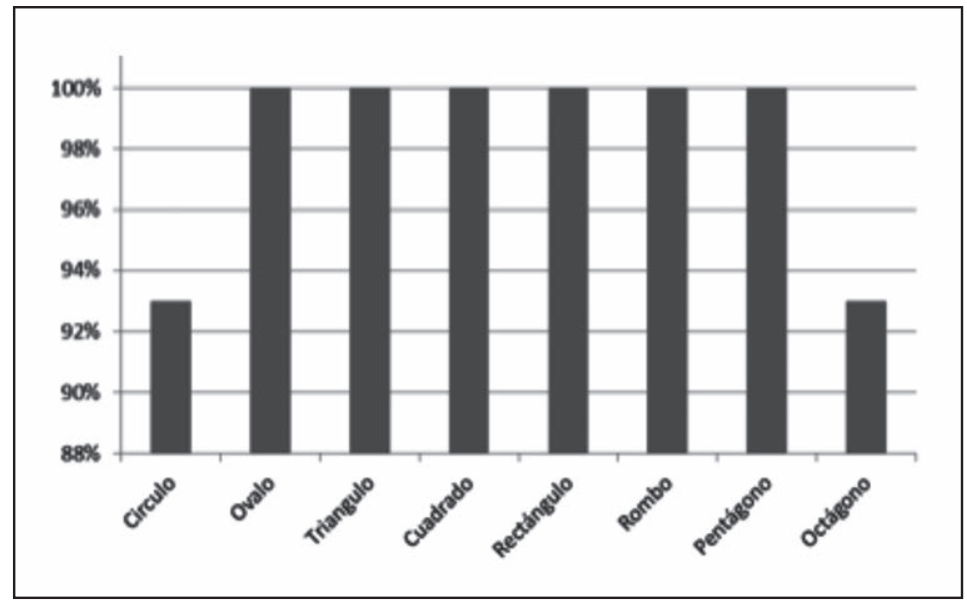

Figura 7. Resultados de reconocimiento.

\section{Conclusiones y recomendaciones}

La implementación de sistemas inteligentes de control sobre manipuladores articulados permite realizar tareas complejas repetitivas a base de un conocimiento experimental sin la necesidad de la intervención de un agente externo de control.

La efectividad en cuanto al reconocimiento de formas depende de la calidad de imagen obtenida tanto en la etapa de pre-procesamiento y acondicionamiento como también de la etapa de extracción de características, conservando la menor cantidad posible 
de ruido ya sea este inducido por el entorno o por los algoritmos de procesamiento.

El porcentaje de aciertos del sistema es del 98,25\%, existiendo problemas de reconocimiento entre el octágono y el círculo.

El momento de $\mathrm{Hu}$ es un buen instrumento matemático para realizar reconocimiento de objetos.

\section{Referencias}

Federación Internacional de Robótica (2016). www.fir.com

Gómez-Allende, D. M. (1994). Reconocimientos de formas y visión artificial. Addison-Wesley Iberoamericana. 